\title{
A Novel Approach for Vehicle License Plate Localization and Recognition
}

\author{
Muhammad H Dashtban \\ Faculty of Electrical and \\ Computer Engineering, \\ University of Kurdistan, \\ Sanandaj, Iran
}

\author{
Zahra Dashtban \\ Faculty of Electrical and Computer \\ Engineering, Shahid Rajee Teacher \\ Training University, Tehran, Iran
}

\author{
Hassan Bevrani \\ Faculty of Electrical and \\ Computer Engineering, University \\ of Kurdistan, Sanandaj, Iran
}

\begin{abstract}
In this paper, a general approach for international vehicle license plate localization and recognition is proposed. A hybrid solution is presented with combining basic machine vision techniques and neural networks. The proposed model consists of three main parts, including localization, segmentation and recognition. In the license plate localization, after some essential preprocessing and finding edges, the 8-connectivity of image background eliminates which helps more appropriately separating of main image objects from the cluttered backgrounds. Then, it is tried to find connected objects with 8-connectivity of the differentiated binary image. The binarization of license plate is based on local binarizing. The proposed recognizing system utilizes the Hough transform, basic morphological operators and Skeletonizing to provide an appropriate input for artificial neural networks. Segment by segment, the input streams into an intelligent error control unit (IECU) which itself is an already trained multi-layer perceptron (MLP) neural network. IECU investigates empty or non-character-inside segments. In case of no error, each segment streams into two already trained MLPs. Each of them singly recognizes either the alphabets or numbers. We show that this approach achieves accuracy over $91 \%$ on localizing vehicle license plate. The image database includes images of various vehicles with different background and slop under varying illumination conditions. The character recognition system correctly recognizes alphabets with probability over $97 \%$ and over $94 \%$ in case of numbers.
\end{abstract}

\section{General Terms}

Machine vision, Image Processing, Neural Network, Algorithm design, implementation.

\section{Keywords}

License plate, LPR, character recognition, Hough transform, neural network recognition, Multi layer perceptron, plate recognition, character segmentation, plate localization, diagonal fill, edge Sobel, OCR, low pass filter Gaussian, TSR.

\section{INTRODUCTION}

Automatic Number Plate Recognition (ANPR) is a transportation intelligent control solution that nowadays inseparably combines with several technologies. This technique in software area is chiefly based on concept and principles of machine vision. However, different scientists utilize these techniques along with various intelligent structures and intuitive synthetic methods. Despite the time nearly three decades from ANPR's emerging has long been passed it still is an active area of research. From a social point of view the need for the intelligent transportation management without human being intervention in different countries throughout the world automatically influenced this field for being long active in literature. Large magnitude of papers within three last decades for ANPR have been demonstrated which shows the importance and the worth of this subject in literature. These researches and corresponding implementations mainly fall into two categories, the vehicle plate localization and the plate character recognition which some literatures are particularly devoted to one of them and some considers both parts. Taking a look beyond the published papers and completed works in license plate identification over the years shows a particular motivation and enthusiastic from researchers. These motivations might address the essence of the subject and corresponding algorithms . In regard to the essence of problem one can classify three factors as main reasons and goals of researches. First is the accuracy which itself is divided into two subclass including accuracy on localization of vehicle license plate and accuracy on recognizing the license plate characters. In this field, especially more complex and robust works have been done in recent years and this is the attribute of advances in technologies which are proportional with increasing accuracy for intelligent agents. The second factor is algorithm time complexity which is significant when the science purpose is implementation. For the APNR, some authors present such robust algorithm but due to the high time complexity they are not applicable in real time systems. However, the authors believe that those approaches could provide a proper pattern for novel hybrid or more simple algorithms. Third factor is adaptability as we expect the intelligent agent, the algorithm or the model has the ability to adapt itself with environment to cope with dynamic outdoor conditions therefore without human being intervention the expected tasks has been done. In the case of license plate identification such conditions address the various lighting, weather, crashed etc. To achieve adaptivity more efforts must be performed. Algorithms with enough generality and high accuracy can perform such tasks that need high adaptive capabilities. Such algorithms by supporting simple and complex conditions in their structural model bring confidence out for human beings. Increasing of confidence to machines is a direct relation with reliability of intelligent agents. Main purpose in this paper is to increase the accuracy along with the reliability. The novel contributions expressly in the current paper are:

1- Developing a novel approach for localizing of vehicle license plate using some preprocessing followed by eliminating 8-connectivity of the background and replacing with corresponding 4-connectivity and so on, 
2- An enhanced method for license plate segmentation based on vertical projected histogram analysis, Hough transform and local thresholding,

3- Address an intelligent error control unit for empty segmented regions using an already trained MLP neural network.

Each part of the proposed general approach is described in detail. Generally, this work divided into three parts including license plate localization, character segmentation and finally character recognition which either has several subparts. The paper is organized as follows: A brief description of some of previous works is demonstrated in section 2. Section 3 includes preliminaries which itself contains two main parts including image database, which is a description of our database properties, and the implementation infrastructure, which nominally lists what are behind this paper, the knowledge base required for implementing the current paper. In section 4 , the algorithms for license plate localization which is followed by license plate segmentation are presented and then, a system based on artificial neural network for controlling error and character recognition is described. In section 5, experimental results are demonstrated.

\section{PAPER REVIEW}

In this section we provide a brief reference source from some of the researchers who previously proposed methods for localization or recognition of license plate. Some of methods are color based which is specially used for license plates with particular background and foreground color. In Ref. [1], the color information of the license plate is used as inputs for training an artificial neural network which itself is then used to find the potential region of license plate. This approach has an accuracy of 0.80 which is not encouraging but it works well for two row license plate. Neural networks for vehicle license plate localization and recognition, namely the Pulse Coupled Neural Networks (PCNN), the Time Delay Neural Networks (TDNN) and the Discrete Time Cellular Neural Networks (DTCNN) are proposed in [2-4]. It is reported that DTCNNs over test data had accuracy of 0.85 , while these approaches often tolerates intensive time complexity in localizing license plate. Authors in [5] used Blob coloring for character segmentation then two multi-layer perception neural networks both with the same structure and one hidden layer for recognition of characters and numbers is separately utilized. Various image segmentation techniques for localizing license plate are proposed in literature. These methods usually use a threshold using which the pixels of image classified into two groups, values below the threshold and above it. For automatically choosing of an appropriate threshold besides the conventional ones, several methods such as classification error [10], inter-class variance [5] and information entropy [6-9] have been proposed. Scale invariant feature transform (SIFT) is another method that is robust and reliable which is used to extract key points from images, those are invariant to rotation and scaling from which is mostly used in object tracking and person authorization. The SIFT is used by [11] for localizing license plate and yield recognition rate .84 . Template matching is a widely approach especially for license plate recognition $[12,13]$. Researchers often should build proper image templates for license plate object like characters, symbols or numbers and then utilize it with a matching algorithm.
Authors in [14] proposed a method for character segmentation in license plate in a multi-national environment with format independent recognition. Extensibility of this method is appropriate but as the method for character segmentation does not use recognition information it is possible to produce false segmented characters, numbers, etc. Many of segmentation approaches for license plate are based on projection. Authors In [15-18] have used vertical, projection. In [19], both vertical and horizontal projection has been used. Generally color or gradient of plate will be projected vertically then in histogram of projection results, the local minimals called "valleys" are assumed as the spaces between characters so the valleys will be used for plate character segmentation. This approach does not work on such vertical projected histogram when there has been a overlap which could be because of bad thresholding or noise or even drop of rains. Besides for histogram with a lot of local minimal it is too hard to make a decision; hence, a deeper analysis and proper preprocessing on license plate can suitably affect the results. Furthermore, the issue of skewed images is a problem for most of segmentation result which should be solved. Blob coloring [20] is another simple and robust technique which some authors such as [21] put it in use to locate characters in the license. Moreover some authors [22] utilized eight-connected version of blob coloring only as a part of license plate localizing procedure. A wavelet transform based approach in [23, 24] have been used. Although these wavelet methods perform well in localization process which finds multiple plates with different orientation angles, but in some cases which the taken image is too far or too near, it might fails. Besides these approaches, several other approaches are proposed in literature which we have not provided herein.

\section{PRELIMINARIES}

What forms the infrastructure of knowledge base in the present paper as well as our image database properties, are demonstrated within two following parts.

\subsection{Infrastructure of Implementation}

This work has been done by sequencing or combining several base techniques which are well known in literature. It seems to be worthy to imply those as an individual part in this paper. The base applied methods in our implementation are as follows:

1- Hough transform

2- Gaussian high/low pass filter

3- Histogram equalization

4- Otsu's method

5- Interpolation

6- Edge detector algorithms

a- Canny

b- Sobel

c- Prewitt

7- Morphological operations

a- Dilation

b- Erosion

c- Tophat

d- Bothat

e- Skeletonizing

8- Neural network

a- Multilayer perceptron

b- Gradient descent learning method

c- Levenberg-Marquardt method 
The methods are stated in this part just nominally, however more details exist in literature which for some, number of valuable works is given in the references.

\subsection{Image Database}

The database for the current study is collected from different places such as public roads, Passages, garages; streets, parking, etc; and some of them are gathered from World Wide Web. After all, the database of our experiment in regards with our need includes the following properties:

1- Varying lighting conditions

2- Different backgrounds (in some instances more than one car but the image only contains one complete license plate)

3- Some taken far from vehicle and some near with.

4- Variable slope

5- Smoothed image

6- Desired ones (more used for training and analyzing )

7- Different plate languages (we test it on two languages, the English and the Persian)

The database is collected in terms of the above properties. Since the automatic vehicle identification system should work in real world, it is tried to involve most of available scenarios and test the proposed method against them.

\section{PROPOSED ALGORITHM}

The proposed algorithm generally is divided into three main parts, including the license plate localization, character segmentation and character recognition. The localized plate, after first part will be used in segmentation system, then character segmentation provide an appropriate output for the recognition system which is an intelligent artificial neural networks based unit. Several algorithmic models are presented for each part in detail.

\subsection{Image Plate Localization}

In order to have a reliable localization of license plate in real dynamic environment, several steps and several choices are required. To have the approach applicable on real time systems in different stages, we usually utilize those choices which have a better time complexity. Corresponding block diagram of total steps of the algorithm in this part is shown in Fig. 1. This part nominally includes the following steps:

1- Noise alleviation

2- Changing color space

3- Intensity dynamic range modification

4- Edge detection

5- Separating objects from background

6- Finding connected component

7- Candidate selection

\subsubsection{Noise alleviation}

Since, images have taken in different conditions that some of them might contain noises, shadows and etc. a proper preprocessing is needed to lessen the effect of noises and adjust image intensity. This task is done in two phases:

a- Intensity uniformization and noise reduction using Gaussian filter before color space transformation,

b- Intensity dynamic range modification after changing color space over gray image.

In the first step, in order to correct image intensity in case of some heterogeneous illumination distribution such as existence of shadow or shadiness on some parts of license plate which significantly affect the localization and the segmentation process, the authors utilize conventional Gaussian low pass filter. This filter additionally alleviates noise affect by attenuating high frequency signals which causes an image to be smoothed.

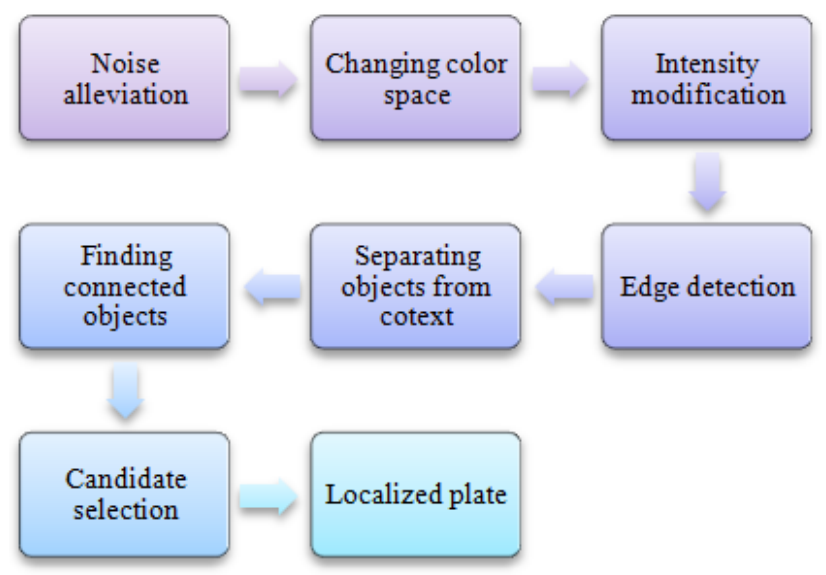

Figure 1. Image plate localization process

General notation for Gaussian low pass filter in two-dimensional image is as follows:

$$
G(x, y)=\frac{1}{2 \pi \sigma^{2}} e^{-\frac{x^{2}+y^{2}}{2 \sigma^{2}}}
$$

where, the $\sigma$ is the standard deviation of Gaussian distribution. The $\mathrm{x}$ and $\mathrm{y}$ are the distances from origin in the horizontal and vertical axis, respectively.

\subsubsection{Changing color space}

Next, the RGB color space is transformed into gray space using the conventional formula (2).

$\mathrm{G}(\mathrm{r})=0.2989 * \operatorname{Red}+0.5870 *$ Green $+0.1140 *$ Blue

\subsubsection{Intensity modification}

In the third phase, for dynamic range modification and somehow sharpening in order to have a better edge detecting in follow up, the conventional histogram equalization is utilized to increase the intensity level range. Sub region histogram equalization is another approach with higher time complexity proposed by some authors [25]. This approach could yield more robust equalization, as well as an appropriate sharpening.

\subsubsection{Edge detection}

In the stage 4 , the vertical Sobel operator is utilized for edge detection. It is important to take an account into the fact that although it is possible to utilize more robust algorithm for edge detection like Canny's method, but since we do not need all of the edges within image, so using Sobel or Prewitt is just enough.

\subsubsection{Separating objects from context}

In next stage, we intuitively utilize diagonal fill to convert image 8-connectivities into corresponding 4-connectivities. An instance of how it works demonstrated in figure 2 . 


\begin{tabular}{|l|l|l|}
\hline 0 & 1 & 0 \\
\hline 1 & 0 & 0 \\
\hline 0 & 0 & 1 \\
\hline
\end{tabular}$\quad$ becomes $\quad$\begin{tabular}{|l|l|l|}
\hline 0 & 1 & 0 \\
\hline 1 & 1 & 0 \\
\hline 0 & 0 & 1 \\
\hline
\end{tabular}

Figure 2. Diagonal fill for eliminating 8-connectivity

It is noteworthy that this approach is only used for enhancement in results so that an improvement over $10 \%$ in localizing license plates is achieved.

\subsubsection{Finding connected component}

Now we try to find the connected objects. The connected objects are investigated with using 8 and 4-ary connectivity. However, only 8-connected objects contain the desired regions, so we should label these connected objects.

\subsubsection{Candidate selection}

As already we have labeled regions, now the candidates for license plate should be selected and then the most similar region to the plate as an output of algorithm must be chosen. Many authors had done this part by investigating geometric features of the extracted regions such as aspect ratio, area and so on. Here, a similar but more efficient method is utilized. It is found out that if the region has been sorted and if the license plate has been among the extracted region, then the region containing license plate is within the four biggest regions. Using this fact, we first sort the extracted regions and mark the four biggest ones as candidates, then we concentrate on the geometric properties only of these regions. Some instances in case of separating four biggest regions have been demonstrated in the experimental result section. Investigating such geometrical properties over our database show that ratio of length to with of the plate region is within the range of 2.2 to 5.7. Furthermore, the length of the plates is more that 60 pixels and the width is more than 15 pixels long, additionally the pixels width is smaller than 90 and the length is smaller than 285 that of images scaled to size $400 * \mathrm{X}$. Here $\mathrm{X}$ is the second dimension of input images preserving the image aspect ratio. Taking some conditions or parameters into the account might slightly improve the result but these slight improvements cause the overall result to be more admissible. In brief, we concentrate on four issues to select an appropriate candidate among four candidate regions.

$$
\begin{aligned}
& \text { a- Area } \\
& \text { b- Ratio of length to width } \\
& \text { c- Range of length and width } \\
& \text { d- Region intensity }
\end{aligned}
$$

The area is used when selecting 4 regions among all extracted region, then other cases will be investigated except the last part. For similar cases, the only issue that can be used is intensity. The proposed algorithm should apply step by step. it means that when only one region has been selected after part $b$ then no need to inspect more. We get output from each part and check out whether there is only one region or not.

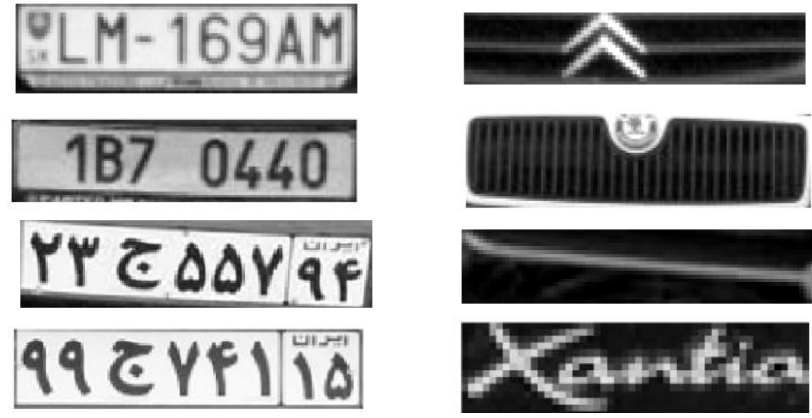

Figure 3. Plate candidate selection from two rectangular regions that are geometrically quite similar

In some cases, two of regions are geometrically quite similar. In such cases we have no way except using the license plate intensity to recognize the right rectangular region. In figure 3 you can see such similarity that we implied, while a significant distinctive exists in their histograms of intensities.

\subsection{Character Segmentation}

After localizing plate in the image, several approaches for character segmentation can be used. Herein we propose a multistage model with a safe confidence level which is able to perform well confronting various situations, and it can be used specially for plate character segmentation. The first part consists of 4 primary operations as follows.

$$
\begin{array}{ll}
\text { 1- } & \text { Improvement } \\
2- & \text { Rotation } \\
3- & \text { Binarization } \\
4- & \text { Segmentation } \\
\text { 5- } & \text { Preparation }
\end{array}
$$

It is important to note that the binary components from previous part are not as appropriate as they can be used for character segmentation. Hence, after localizing the plate in the image, we can extract the plate from the gray image again, and processes that instead.

\subsubsection{Improvement}

The Improvement includes two subparts as

$$
\begin{aligned}
& \text { a- Noise reduction, } \\
& \text { b- Contrast enhancement. }
\end{aligned}
$$

We utilize a 2-d median filter [27] to remove effect of some unwanted noises. Besides the contrast enhancement in case of intensity distribution problem for intensity images with low or poor dynamic range, the histogram equalization is utilized. Using histogram equalization could be more appropriate especially when local binarization methods are supposed afterward to be used.

\subsubsection{Rotation}

Since images might have been taken in different angles, we need to estimate the skew angle of the image and rotate it in order to have a right plate. Although some authors utilize Hough transform directly to segment the characters, but in different situations it seems not work well as for the hard, smoothed or 
line noisy conditions. Here the Hough transform with Canny algorithm is used only to find the greatest line within the plate image and determine the plate slope. Using Hough with some other edge detection algorithm like Sobel or Prewitt does not guaranteed to find the appropriate line in car plates under varying illumination conditions especially for some very smooth plates does not work well. Consequently, we utilize Canny line detection algorithm and perform Hough transform to find the longest line in the plate image. The slope of the line is then used to rotate the plate. Some illustrations for finding longest line are demonstrated in figure 4.

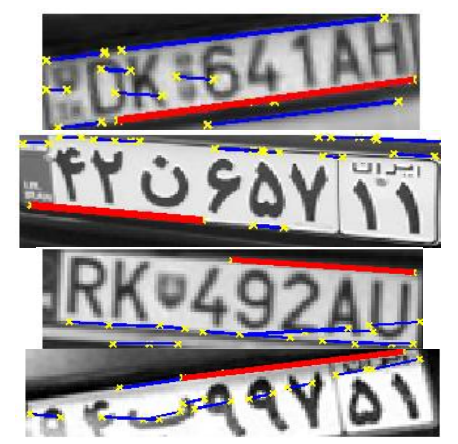

Figure 4. Finding lines using Hough transform (red lines are the biggest line in each license plate)

\subsubsection{Binarization}

The third phase is binarization, which we need an appropriate threshold. Several methods for choosing fine threshold are available which can be divided into two main categories: finding a global threshold and local thresholds. However, using a global threshold due to the intensity variance in images does not guarantee a proper result even using such iterative method like Otsu's method [26]. While in many cases, Otsu's method due to a good preprocessing does perform well. Here, for a general approach with a good confidence level, an adaptive local thresholding along with Otsu's method is used.

a- Divide the image plate horizontally into three unequal parts $20 \%, 80 \%$ and $20 \%$, respectively

b- Divide the image plate vertically in five unequal parts $15 \%$, $20 \%, 30 \%, 20 \%$ and $15 \%$

c- Calculate threshold using Otsu's method for each block.

d-Binarize the plate image using the threshold matrix obtained in (c).

The reason of such unequal division is that the plate boundaries obtained from previous phase usually comes thicken and noisy due to the shadowing and some illumination condition. This affects the tuning of general thresholding.

\subsubsection{Segmentation}

This phase deals with segmenting the binary image plate into characters, for this purpose several works have been done. Herein, an enhanced approach for character segmentation is proposed. Some of authors such as [28, 29] have previously utilized histogram based segmentation which does not almost pay attention to the very close or overlapped characters in some plates. Such overlaps could be from a stain, smudge or generally noises. We propose a simple and modified approach which is easy to implement with which we got proper results. This idea is mainly based on binary vertical-projection histogram analysis.
Using these techniques without proper preprocessing might not give good results. Several issues exist when a histogram based method for segmentation is utilized. There are several closed local minimals in the histogram of some plates which are usually too noisy or too smoothed. Overlap is another issue. Noisy objects like stain or some lighting conditions produce overlaps in the histogram of plates. In these conditions, it is hard to segment the plate characters in terms of the histogram analysis. Here, it is tried to include these conditions in proposed method. Some morphological operators are used to make the analysis simpler and more reliable. The approach can be summarized as follows.

i. Eliminate main plate boundary which is appear usually dark around the plates (this can be easily done using a simple horizontally-projected histogram analysis which computationally makes no overhead as one time we do projection and use that throughout the algorithm).

ii. Erode the image using somehow big structure element (the recommended structure element radius should be something bigger than the distance of the two near minimal in the middle of vertically-projected histogram of image. Because in some countries some characters appear vertically thinner in the middle of plate and so we must take those into the account)

iii. Make a histogram out of vertically projected pixels of the binary image obtained from part 2.

Here, distinct mass can be seen obviously in the histogram of the plate. An example is shown in Fig.6. The purpose is selecting a proper point within each pair of adjacent mass, a reliable point that can separate appropriately two adjacent characters in the plate. To find the point, the vertical-projected histogram of the plate before erosion is utilized. In this histogram, minimum points that are located within gaps of previous histogram are the objectives. Since, In some histograms an strong minimum for each gap, does not find, so multiple local minimal are utilized. Desired local minimals usually have poor intensity rather than adjacent points. Hence, only the local minimals with the height less than $80 \%$ of average intensity values are investigated.

1- Find the local minimal smaller than the 0.80 of the average height of the vertically-projected histogram (the heights within the gap only),

2-Try to find two points that have the longest immediate distance from its adjacent peak,

3-Obtain the point located between the points in step 2 and store it as a delimiter.

4- Repeat for each gap

Finally, the characters in the plate will separate from each other using the delimiters.

\subsubsection{Preparation}

Fifth phase is preparing segmented blocks for neural networks. In this part we do some preprocessing to enhance the result of neural network in the next phase. Processing herein is as below:

1 - Erode the segments using a 2 by 2 square mask,

2-Dilate the segments from part 1 using a disk shape mask with radius of amplitude 2,

3- Skeletonize the segments,

4- Resize each segment to a 30 by 20 matrix.

Note that we first perform morphological erosion to eliminate some small block that is usually noise and then we perform the morphological dilation to fill some holes. The other thing that is worth to be noted is instead of using the morphological opening, 
erosion and dilation are separately applied to provide different structure elements for each one.

\subsection{Character Recognition}

Several applications have been implemented using artificial neural networks over the years. Different types of neural network are also being used for character recognition in license plate. Authors in [30-32] utilized an MLP with a feed forward neural network for recognizing characters. A feed forward neural network model is presented in Fig.5.

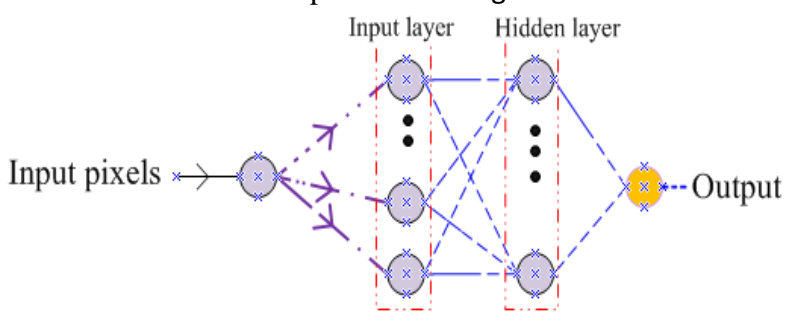

Figure 5. Feed forward neural network.

A two layer MLP containing 24 input, 15 hidden and 36 output neurons are used in [31] for recognizing 36 characters (including letters and numbers). The achieved encouraging accuracy is about $98 \%$ over a large database. In [32], a comparison between Hopfield and MLP presented which achieved the accuracy of $87 \%$ in case of Hopfield network.

Here, for increasing accuracy in recognition, two independent MLPs are utilized, one for letters and one for numbers. Additionally, one other MLP has been trained and utilized as an intelligent control error unit in order to control the empty segments. Empty segments mean segments containing no character such as a stain or blocks adhere to plates and other noisy segments which were segmented correctly in previous part (but it has no character inside).

The Levenberg-Marquardt method [33, 34] is used, which is one of the numerical optimization methods and can successfully train the MLP neural networks. All of the MLPs used in this paper have three (input, hidden and output) layers. Using two separate MLP for letters and numbers does work fine and yield an accuracy over $94 \%$ and $97 \%$ for numbers and alphabets, respectively. It is worth to note that since MLP neural network initially are sensitive to noise which significantly affect the results, noisy numbers and characters have been utilized for training neural networks. Note that these results come from our implementation over 200 various license plates. The MLP network structure for numbers contains 24 inputs, 12 hidden and 24 output neurons. Moreover the MLP of numbers contains 10 inputs, 8 hidden and 10 output neurons.

\section{Experimental Results}

The method described in section 4 is applied to localize and recognize the license plates within a dataset of 200 various images which license plates characters were from two different languages, the Persian and the English. The method achieved accuracy over $91 \%$ for localizing plates. The recognition system implemented by neural networks after segmentation of characters in image plate identify alphabets and numbers separably and achieve an accuracy over $97 \%$ and $94 \%$ respectively for each. In Fig. 6, some examples for selecting the four biggest objects from labeled image are demonstrated. A comparison between the binarization methods proposed in section 4.2.3 and Otsu method for a particular image is demonstrated in Fig.7. In Fig.8, characters in the plate are connected to their above boundary after global thresholding which could affect segmentation and recognition process. Horizontal and vertical histograms of license plate of figure 8 are demonstrated in Fig. 9 and 10, respectively. In figure 11, vertical histogram of plate after erosion and boundary elimination in regard with the proposed segmentation method in section 4.2.4 is presented. Character boundaries for segmentation in the histogram are obvious. Furthermore, the third mass from the left within the histogram of fig. 10 will be segmented by our method but by controlling empty segments by an already trained MLP neural network, this would not produce an output.

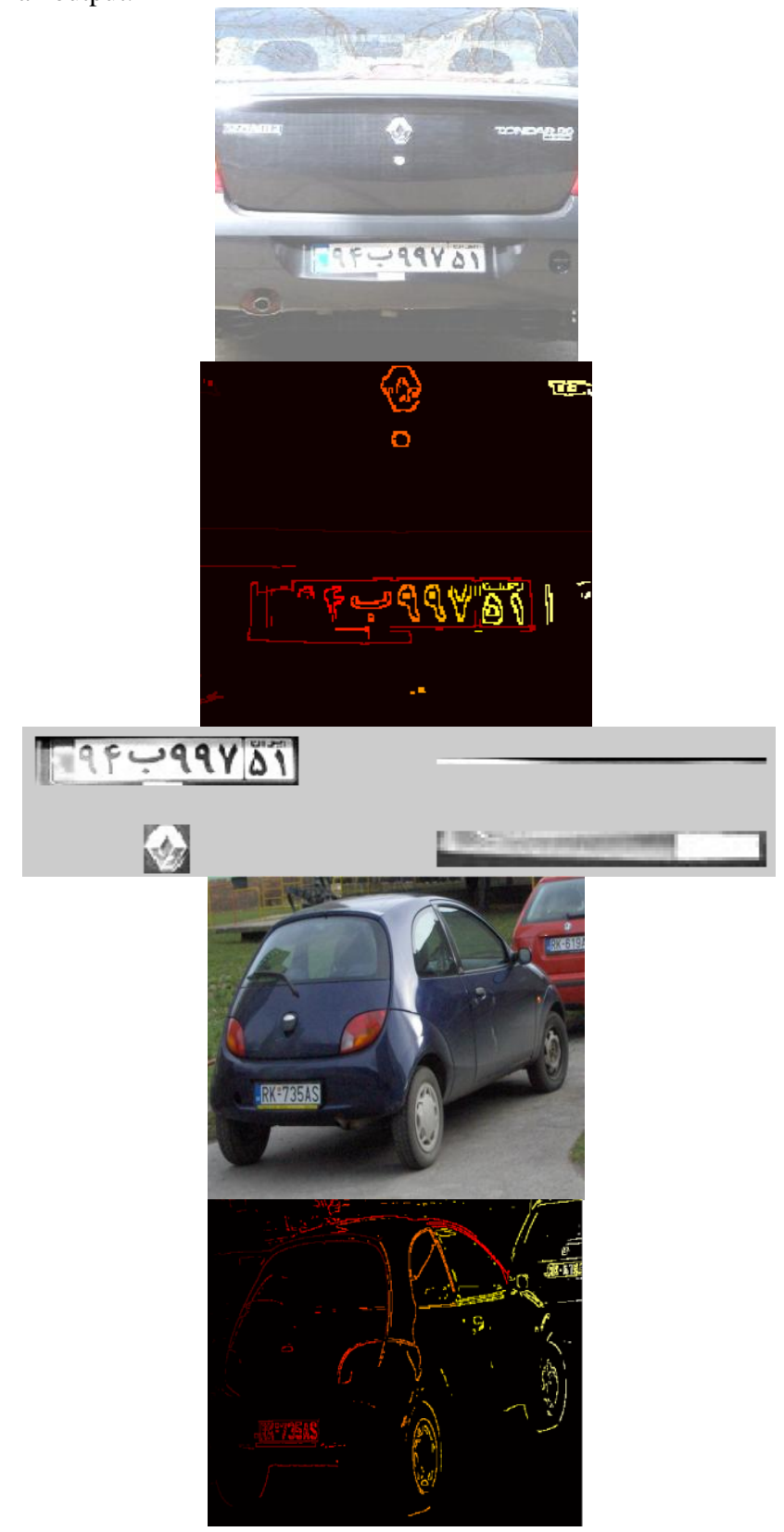




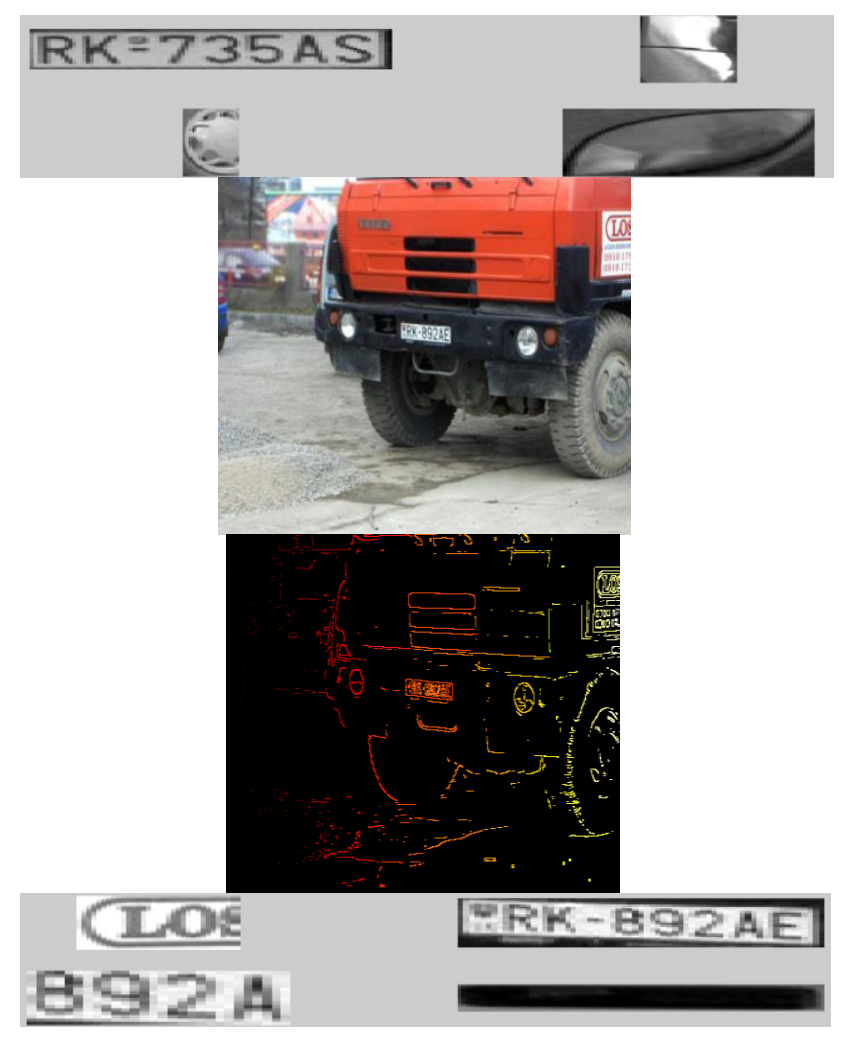

Figure 6. License plate localization. Up left is the input image, up right is the labeled image from 8-connected objects, bottom is the image of the four biggest objects

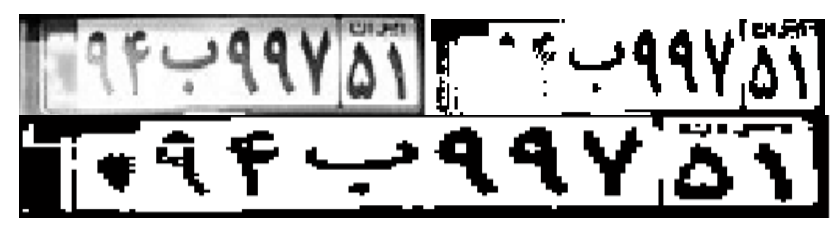

Figure 7. License plate binarization. Up left is the gray plate, up right binarized using Otsu method, bottom binarized using proposed method in section 4.2.3

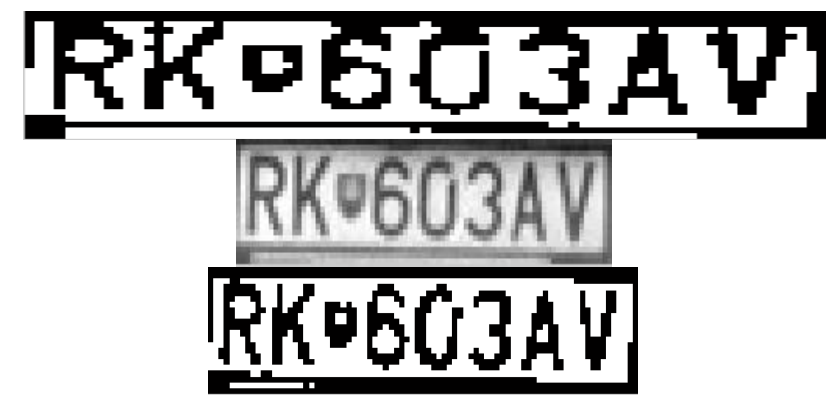

Figure 8. Local binarization against noises, up is binarized using global thresholding using Otsu method, bottom right is local binarized, bottom left is the input gray image

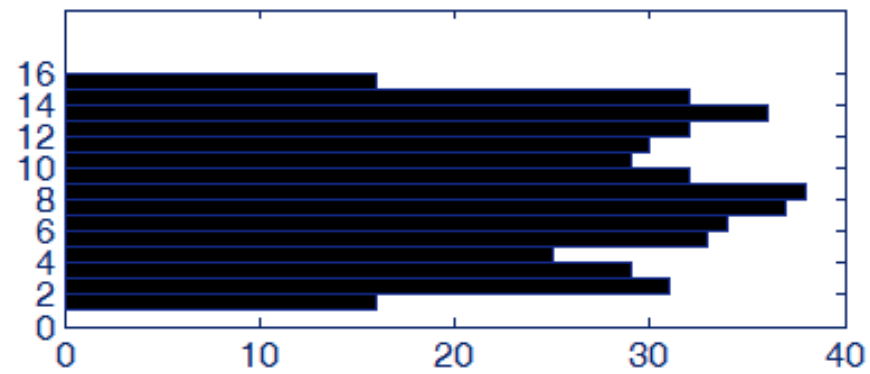

Figure 9. Horizontally projected pixels for plate in fig.8

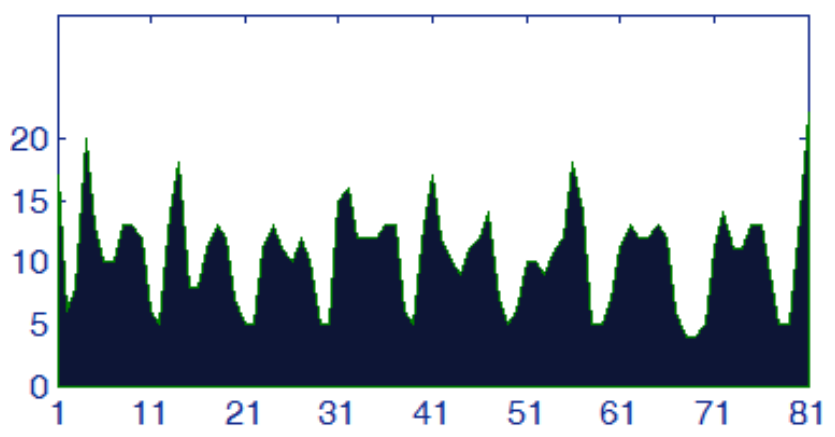

Figure 10. Vertically projected histogram of plate in fig.8

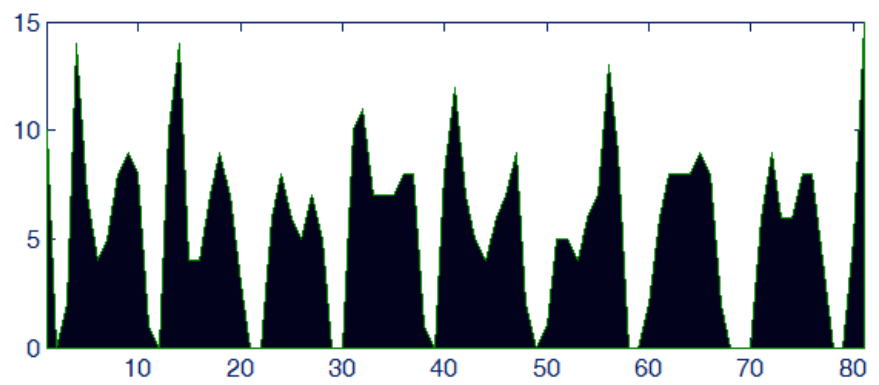

Figure 11. Vertically projected histogram of plate after removing horizontal boundary and eroding proposed in section 4.2.4

\section{CONCLUSION}

In this paper, a general algorithmic model is proposed for vehicle plate identification. This is not a color based approach and due to the special processing, the method can be used in car screening systems of several countries worldwide. The proposed algorithm generally consist of three main parts, First the license plate detection model which has several steps. For enhancing overall results it converts 8-connectivity of background and finds desired connected components of input image with 8connectivity. In the first step we had some special preprocessing which decay such issues as shadow or shadiness which are the result of various illumination conditions. This issue is done by using Gaussian low pass filter and histogram equalization for sharpening and increasing image intensity dynamic range. After extracting the appropriate object among the four biggest 8connected objects, the character segmentation system utilized the Hough transform to find the longest line within the extracted boundary. This line was then used for its angle as the angle of 
the plate and rotation problem in skewed car plates using the obtained angle would be solved. Local binarization is used and then some basic morphological operations for enhancing the extracted plate are utilized. Then, a vertical projected histogram based technique in regard with the valleys is used to find space between characters. Some morphological operations to overcome overlapped characters are successfully utilized. Then, the system provided a scaled version of each segment using such techniques such as skeletonization for the next step. Inputs herein are segments having size 30 by 20 that streamed into an intelligent error control unit (IECU) which contains a simple and already trained multi-layer perceptron neural network which decides whether the input segment contains error or not. In regard with the result of IECU the processed inputs segment by segment streamed into two other artificial neural networks which were Multi-Layer Perceptron neural network. To achieve a better result in recognition of segments each of neural networks designed especially for either numbers or characters and processed its inputs singly and independently however both had the same internal structure. To implement and simulate the results we have created our own database of cars, plates, characters with such conditions implied in part 2.2 which had some various properties such as varying illumination conditions, different backgrounds, skewed images and etc. The experimental results obtained by applying the proposed method on a test database that contains 200 various vehicle images with scaled size of width 400 by preserving length X. Experimental results showed that the proposed method performs well with rounded accuracy of 0.91 for localizing vehicle license plate and correct recognition rate for the successfully localized license plates in case of character achieved an accuracy of 0.97 and .94 for alphabets and numbers, respectively.

\section{REFERENCES}

[1] Satadal Saha, Subhadip Basu, Mita Nasipuri, Dipak Kr. Basu, "Localization of License Plates from Surveillance Camera Images: A Color Feature Based ANN Approach", International Journal of Computer Applications, 2010, Volume 1 - No. 23

[2] M. H. Brugge, J. H. Stevens, J. A. G. Nijhuis, L. Spaanenburg, "License Plate Recognition Using DTCNNs", in proc. IEEE lnt. Workshop on Cellular Neural Networks and their Applications, 1998, pp. 212-217.

[3] Mario I. Chacon M., Alejandro Zimmerman S., "License Plate Location Based on a Dynamic PCNN Scheme", in proc. Int. Joint Conf. on Neural Networks, vol. 2, 2003, pp. $1195-1200$.

[4] K. K. Kim, K. I. Kim, J. B. Kim, H. J. Kim, "LearningBased Approach, for License Plate Recognition", in proc. IEEE Signal Processing Society Workshop, Neural Networks for Signal Processing, Vol. 2, 2000, pp. 614 623.

[5] K. I. Kim, K. Jung, J. H. Kim, Color texture-based object detection: an application to license plate localization, in: S.W. Lee, A. Verri (Eds.), Lecture Notes on Computer Science, vol. 2388, Springer, New York, 2002, pp. 293 309.
[6] S. Draghici, A neural network based artificial vision system for license plate recognition, International Journal Neural Systems 8 (1997) 113-126.

[7] R. Parisi, E. D. Di Claudio, G. Lucarelli, G. Orlandi, Car plate recognition y neural networks and image processing, IEEE International Symposium on Circuits and Systems 3 (1998) 195-198.

[8] Gang Li, Ruili Zeng, Ling Lin, Research on vehicle license plate location based on neural networks, in: First International Conference on Innovative Computing, Information and Control, 2006.

[9] X. Shi, W. Zhao, Y. Shen, Automatic license plate recognition system based on color image processing, in: $\mathrm{O}$. Gervasi et al. (Eds.), Lecture Notes on Computer Science, vol. 3483, Springer, New York, 2005, pp. 1159-1168.

[10] E. R. Lee, P.K. Kim, H. J. Kim, Automatic recognition of a car license plate using color image processing, in: IEEE International Conference on Image Processing,Austin, Texas, 1994, pp. 301-305.

[11] M. Zahedi ,S. M. Salehi. License Plate Recognition System Based on SIFT Features, Procedia Computer Science,3,2011

[12] M. A. Ko, Y. M. Kim, License plate surveillance system using weighted template matching, in: Proceedings of Applied Imagery Pattern Recognition Workshop, 2003, pp. 269-274.

[13] Y. P. Huang, S.Y. Lai, W.P. Chuang, A template-based model for license plate recognition, in: Proceedings of the 2004 IEEE International Conference on Networking, Sensing \& Control, vol. 2, 2004, pp. 737-742.

[14] V. Shapiro, G. Gluhchev, Multinational license plate recognition system: segmentation and classification, in: ICPR, vol. 4, 2004, pp. 352-355.

[15] H. A. Hegt, R. J. De la Haye, N.A. Khan, A high performance license plate recognition system, in: Proceedings of IEEE International Conference on System, Man and Cybernetics, vol. 5, 1998, pp. 4357-4362.

[16] M. M. Shidore, S. P. Narote, Number Plate Recognition for Indian Vehicles, International Journal of Computer Science and Network Security, VOL.11 No.2, Feb. 2011

[17] J. Barroso, A. Rafel, E. L. Dagless, J. BulasCruz, Number plate reading using computer vision, IEEE Int. Symp. Ind. Electron. ISIE'97 3 (1997) 761-766.

[18] N. Xin, S. Lansun, Research on license plate recognition technology. Measurement \& Control Technology, 1999, pp. 14-17 (in Chinese).

[19] K. M. Hung,C.T. Hsieh, Real-Time Mobile Vehicle License Plate Detection and Recognition, Tamkang Journal of Science and Engineering, Vol. 13, No. 4, pp. 433_442 (2010)

[20] D. H. Ballard, C. M. Brown, Computer Vision, Pages 151152, Prentice Hall, New Jersey, 1982. 
[21] H. E. Kocer, K. K. Cevik, Artificial neural networks based vehicle license plate recognition, Procedia Computer Science, Volume 3, 2011, Pages 1033-1037

[22] F. Kahraman, B. Kurt,M. Gökmen, License Plate Character Segmentation Based on the Gabor Transform and Vector Quantization,ISCIS 2003,LNCS 2869,pp. 381-388,2003.

[23] Y. Cui, Q. Huang, "Extracting characters of license plates from video sequences", Machine Vision and Applications, vol. 10, pp. 308-320, 1998

[24] Y. R. Wang, W. H. Lin, S. J. Horng, A sliding window technique for efficient license localization based on discrete wavelet transform, Expert Systems with Applications, Volume 38, Issue 4, April 2011, Pages 3142-3146

[25] H. Ibrahim, N. S. P. Kong,” Image Sharpening Using SubRegions Histogram Equalization", IEEE T CONSUM ELECTR, 55, 891-895, 2009.

[26] N. Otsu, "A threshold selection method from gray-level histogram, IEEE Transactions on Systems", Man, and Cybernetics 9 (1979) 62-66.

[27] Lim, Jae S., Two-Dimensional Signal and Image Processing, Englewood Cliffs, NJ, Prentice Hall, 1990, pp. 469-476.
[28] C. A. Rahman, A. Radmanesh, A Real Time Vehicle's License Plate Recognition System,Proceedings of the IEEE Conference on Advanced Video and Signal Based Surveillance, 2003.

[29] C. C. Chen, J. W. Hsieh, License Plate Recognition from Low-Quality Videos, VA2007 IAPR Conference on Machine Vision Applications, May 16-18, 2007.

[30] A. Broumandnia, M. Fathy, Application of pattern recognition for Farsi license plate recognition, 2005

[31] J. A. G. Nijhuis, M. H. ter Brugge, K.A. Helmholt, J.P.W. Pluim, L. Spaanenburg,R.S. Venema, M.A. Westenberg, "Car License Plate Recognition with Neural Networks and Fuzzy Logic", in proc.. IEEE International Conference on Neural Networks, vol.5, 1995, pp. 2232-2236.

[32] A. Akoum, B. Daya , P. Chauvet, "two neural networks for license number plates recognition", Journal of Theoretical and Applied Information Technology, 2005 - 2009 JATIT.

[33] Levenberg, K., "A Method for the Solution of Certain Problems in Least Squares," Quart. Appl. Math. Vol. 2, pp 164-168, 1944.

[34] Marquardt, D., "An Algorithm for Least-Squares Estimation of Nonlinear Parameters," SIAM J. Appl. Math. Vol. 11, pp 431-441, 1963. 